\title{
The quality of working impressions for the fabrication of fixed prosthodontics prostheses (crown and bridgework)
}

\author{
Ahmad Syahir Ahmad Zu Saifudin, Fazlieha Kamaruddin, Siti Mariam Ab Ghani \\ Centre for Restorative Dentistry Studies, Faculty of Dentistry, Universiti Teknologi MARA, 40450 \\ Shah Alam, Selangor, Malaysia
}

\author{
Address for correspondence: \\ Dr. Siti Mariam Ab Ghani, \\ Centre for Restorative Dentistry \\ Studies, Faculty of Dentistry, Universiti \\ Teknologi MARA, 40450 Shah Alam, \\ Selangor, Malaysia. \\ E-mail: sitimariam783@salam.uitm. \\ edu.my
}

\begin{abstract}
Objective: The study was carried out to assess the quality of working impressions sent to commercial laboratories for fabrication of fixed prostheses. Materials and Methods: Impressions ( $\mathrm{n}: 200)$ received by four dental laboratories were evaluated by two calibrated examiners. The type of work, tray designs, impression materials and techniques were recorded for each impression. Quality data on crucial details of the tooth preparations, voids at tooth preparations, tears along the finished margin line and flow of materials were documented and each criterion scored either with 2 (Good), 1 (Acceptable) or 0 (Unacceptable). The Chi-square test for independence was done for relationship analysis of the impression defects and different type of impression materials. Results: From 200 examined impressions, $53.9 \%$ were for crown works, 35.9\% bridge works, $1.4 \%$ posts and cores and $8.8 \%$ for other types of work. The impression materials used were polyether (39.5\%) and polyvinyl-siloxane (60.5\%). The two main types of trays were metal stock (48.5\%) and disposable plastic (37.5\%). Impression techniques were monophase one-step technique (50.0\%), putty wash two-steps technique (23.5\%), putty wash one-step technique (15.5\%) and dual phase one-step technique (11.0\%). Overall, $64.5 \%$ of the impressions sent had unacceptable quality ( 0 score recorded for at least one criteria). The proportions of impression defects were significant to the type of impression materials $(P<0.05)$. Conclusion: The percentage of unacceptable impressions $(64.5 \%)$ sent to the laboratories is a serious concern, as it contributes to the inaccuracy of the crown and bridgeworks provided to patients.
\end{abstract}

Key words

Fixed prosthodontics, impressions quality, polyvinyl-siloxane

\section{INTRODUCTION}

An impression is defined as a negative likeness or copy in reverse of the surface of an object. ${ }^{[1]}$ Impression materials in the market can be classified by the chemical type, physical properties, viscosities or by the manufacturers' brand. The widely used classification is according to their elastic properties and chemical type [Figure 1]. Many studies on impression accuracy have been published. ${ }^{[2-4]}$ Majorities were looking at the resultant dies, which the inaccuracy can also be from factors such as the different techniques for making impressions, ${ }^{[4]}$ laboratory handling, pouring stage ${ }^{[5]}$ or the dimensional stability of

\begin{tabular}{|l|l|}
\hline \multicolumn{2}{|c|}{ Access this article online } \\
\hline Quick Response Code: & Website: \\
\cline { 1 - 2 } & www.ejgd.org \\
\cline { 2 - 3 } & \\
\hline
\end{tabular}

the dies. Only few studies reported on the quality of the impressions made clinically. ${ }^{[6]}$

The aim of this study was to evaluate the quality of impressions sent to commercial laboratories for fabrication of fixed prosthodontics prostheses, to describe the frequency of clinically detectable impression errors and to determine possible correlations between various factors.

\section{MATERIALS AND METHODS}

Four dental laboratories were chosen as the research subject for the reason of good distribution of cases accepted by these laboratories. A total of 200 working impressions for fabrication of fixed prostheses were examined between November 2012 and May 2013 (n: 50 for each laboratory). All of the impressions were evaluated immediately upon reaching the laboratories after collected from various dental clinics to avoid any laboratory handling error. The impressions were also numbered prior to evaluations so that the identities of the 


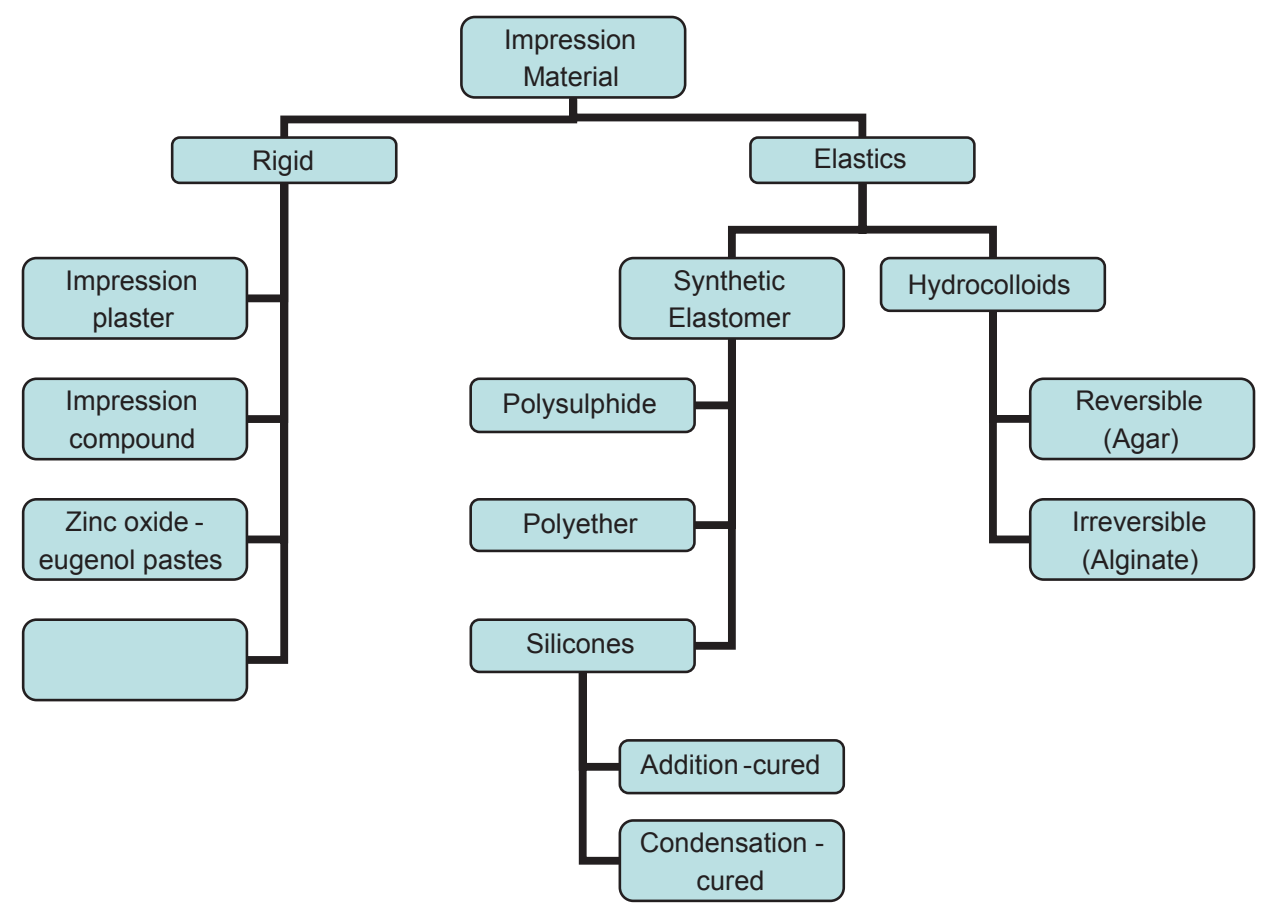

Figure 1: Classification of dental impression materials

dentists were unknown to the examiners in preventing bias evaluations. Two calibrated and independent examiners evaluated the impressions. Inter-operator and intra-operator calibration was done in a pilot study between two Year 5 students and a prosthodontist. The prosthodontist was selected as the standard evaluator and Kappa statistical analysis was performed.

For each impression, the type of work, the impression technique, type of tray, retention method of impressions to tray and the material used were recorded. Data on the quality of impressions were documented, including the crucial fine details of the tooth preparation, voids at the tooth preparation, tears at finished margin line and flow of the impression materials (presence of step/streak). Each criterion evaluated was scored either with 2 (Good), 1 (Acceptable) or 0 (Unacceptable).

The collected data were then entered into SPSS version 20 for further analysis. Descriptive result and Chi-square test for independence for relationship analysis of the impression defects and type of impression materials were performed.

\section{RESULTS}

From the 200 evaluated impressions, it was identified that $53.9 \%$ of the impressions were for crown works, $35.9 \%$ for bridge works, $1.4 \%$ for posts and cores and $8.8 \%$ for other types of prosthodontics works which mainly consist of implant supported fixed prostheses works. The main type of impression materials used by the dental practitioners was polyether (39.5\%) and polyvinyl-siloxane (60.5\%). For impression trays, the 2 main types of trays were metal stock trays $(48.5 \%)$ and disposable plastic trays (37.5\%). As for the impression techniques, majority of the dental practitioners made the impressions using monophase technique (50\%), $23.5 \%$ putty wash two-steps technique, $15.5 \%$ putty wash one-step technique and $11.0 \%$ by dual phase one-step technique. The overall results for general evaluation of impressions were shown in Table 1.

The percentage of impressions that were unable to record the fine detail of tooth preparation according to material was PVS addition-cured silicone (37.2\%), polyether $(28.7 \%)$ and PVS condensation cured silicone $(28.0 \%)$ as shown in Figure 2.

The highest occurrence of unacceptable impression by present of voids at the tooth preparation was polyether (61.2\%), PVS condensation-cured silicone (39.2\%) and PVS addition-cured silicone (33.8\%). The percentage for each material is shown in Figure 3.

For the presence of tears at finished margin line, polyether showed the highest occurrence (32.4\%), PVS addition-cured silicone (12.5\%) and PVS condensation-cured silicone (12.9\%) as shown in Figure 4.

For the presence of flow problem of impression materials, PVS addition-cured silicone showed the highest occurrence (57.4\%), polyether (30.5\%) and PVS condensation-cured silicone $(20.8 \%)$. The occurrence of flow problem in each material is shown in Figure 5. 


\begin{tabular}{|c|c|c|}
\hline $\begin{array}{l}\text { Table 1: General evaluation d } \\
\text { Type of work }\end{array}$ & eac & ssion \\
\hline Crown & 117 & $53.92 \%$ \\
\hline Bridge & 78 & $35.94 \%$ \\
\hline Post and core & 3 & $1.38 \%$ \\
\hline Inlay & o & $0.00 \%$ \\
\hline Onlay & o & $0.00 \%$ \\
\hline Others-Implant & 19 & $8.76 \%$ \\
\hline Total & 217 & $100.00 \%$ \\
\hline \multicolumn{3}{|l|}{ Type of impression material } \\
\hline Alginate & 0 & $0.00 \%$ \\
\hline Polyether & 79 & $39.50 \%$ \\
\hline \multicolumn{3}{|l|}{ Silicone } \\
\hline Addition & 47 & $23.50 \%$ \\
\hline Condensation (putty) & 74 & $37.00 \%$ \\
\hline Others & o & $0.00 \%$ \\
\hline Total & 200 & $100.00 \%$ \\
\hline \multicolumn{3}{|l|}{ Type of tray used } \\
\hline \multicolumn{3}{|l|}{ Stock tray } \\
\hline Disposable plastic tray & 75 & $37.50 \%$ \\
\hline Metal tray & 97 & $48.50 \%$ \\
\hline Others & 0 & $0.00 \%$ \\
\hline \multicolumn{3}{|l|}{ Custom-made tray } \\
\hline Thermoplastic resin & 9 & $4.50 \%$ \\
\hline Light-polimerized acrylic resin & 19 & $9.50 \%$ \\
\hline Others & o & $0.00 \%$ \\
\hline Total & 200 & $100.00 \%$ \\
\hline \multicolumn{3}{|l|}{ Impression technique } \\
\hline \multicolumn{3}{|l|}{ Putty/Wash technique } \\
\hline One step & 31 & $15.50 \%$ \\
\hline Two steps & 47 & $23.50 \%$ \\
\hline Dual phase, single step technique & 22 & $11.00 \%$ \\
\hline Monophase, one step technique & 100 & $50.00 \%$ \\
\hline Total & 200 & $100.00 \%$ \\
\hline
\end{tabular}

The status of an overall unacceptable impression was given to any impression that record unacceptable status to any of the four criteria in Table 2. Upon evaluation of the impressions according to the criterion, 64.5\% (n: 129/200) of the impressions sent to the four laboratories had unacceptable qualities. The percentage was reported as overall and not specifically according the any laboratories.

Further statistical analysis using Chi-square test of independence was done on each quality criteria and significant relationship was noted $(P<0.05)$ on the proportions of impression defects to the type of impression materials. The quality criteria that were significant were the present of voids, tears at preparation margin and inadequate flow of the impression material.

\section{DISCUSSION}

The results of this study indicated that the most commonly used materials by the general practitioners for their crown and bridgeworks were elastomeric

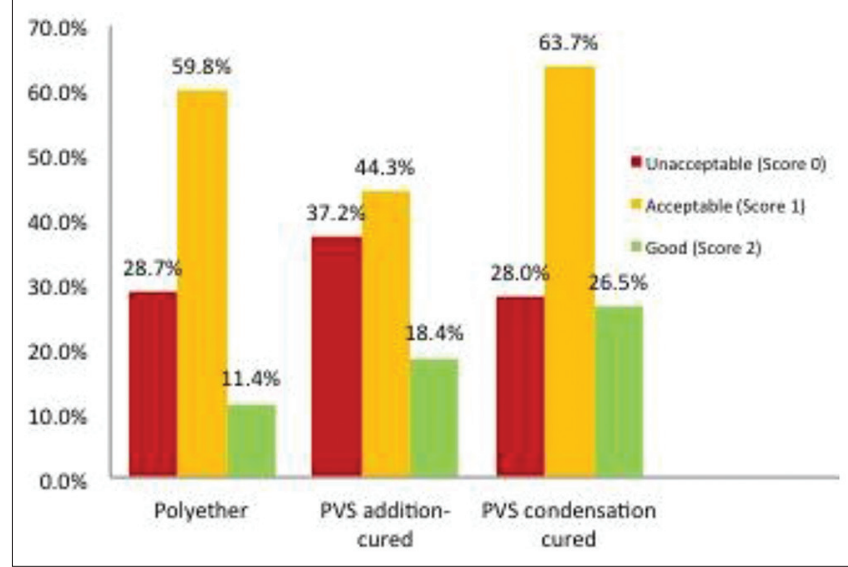

Figure 2: Crucial fine detail of tooth preparation

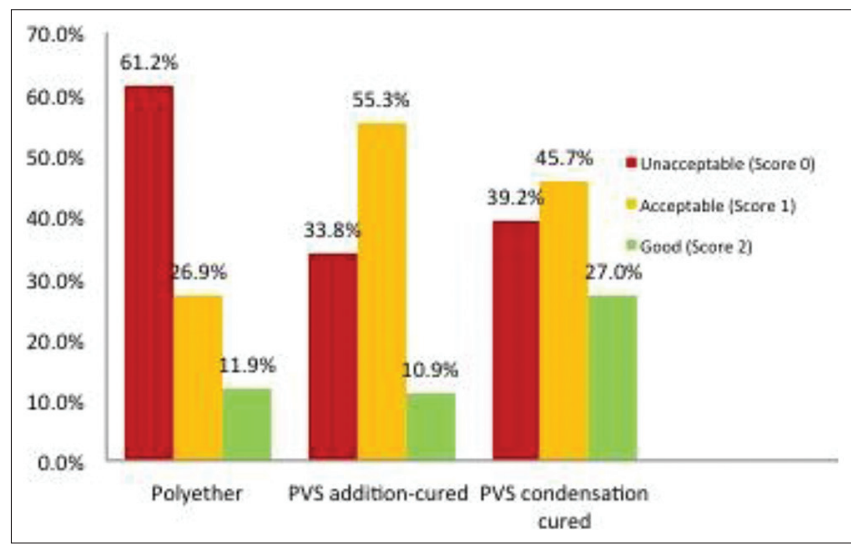

Figure 3: Voids (air bubbles) at the tooth preparation

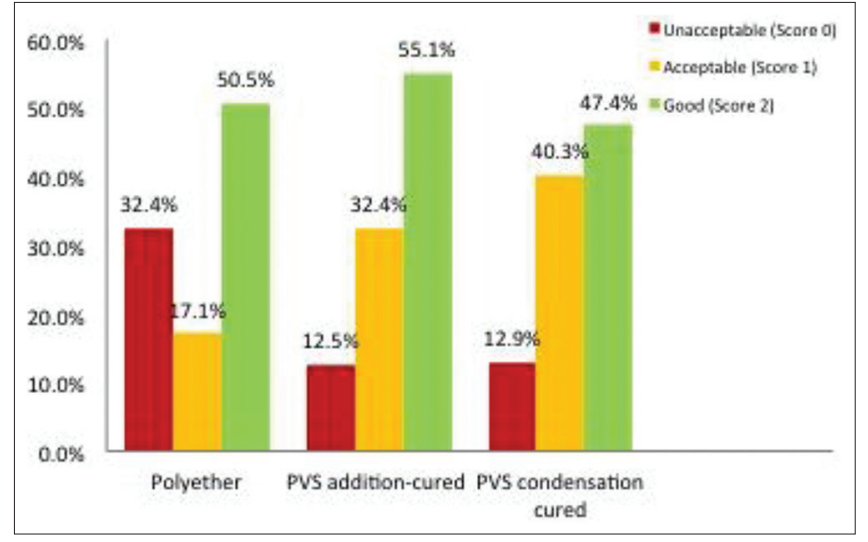

Figure 4: Tears at finished margin of tooth preparation

impression material. These practices were concurrent with the practice worldwide. Both polyether and polyvinyl-siloxane impression materials were known to have the ability to record fine details, minimal dimensional change after setting, moderately short working and setting time and excellent recovery from deformation on removal. ${ }^{[7]}$

In order to increase the accuracy of working impressions, dimensional stability of an impression tray is also a 
contributing factor. Trays should have good stability along a period of time and does not portray any permanent deformation between impression taking and pouring stage. ${ }^{[5]}$ Metal tray are rigid and will prevent distortion upon usage with high viscosity impression material such as putty ${ }^{[8]}$ while the custom-made tray will provide uniform space for a uniform polymerization shrinkage of impression material. This study reported that $48.5 \%$ uses metal stock trays and only $14 \%$ uses custom-made trays for making the working impressions.

There are different impression techniques used with elastomeric impression material with each technique has their advantages and disadvantages. Putty-wash technique was reported that it may eliminate the need of custom-made tray, therefore it coincided with $48.5 \%$ of the impressions were taken using metal stock trays in this study. Few researchers believed that the 2-step putty/wash technique is superior as it minimizes the amount of alcohol byproduct, eliminate the polymerization shrinkage from the first impression step (putty) and thereby retains the dimensional stability of the impression. ${ }^{[3]}$

The apprehension finding from the present study was that 129 out of 200 impressions (64.5\%) were evaluated as unacceptable working impressions. An impression with one criteria in Table 2 scored as unacceptable was recorded as an overall unacceptable impression because lacked on one of these criteria can cause major inaccuracy of the fabricated fixed prostheses. The clinicians' identities were not recorded due to ethical issues, although this information could be valuable to determine the impression quality according to the group of clinicians; students, general practitioners or specialists. There are many reasons for the high incidence of unacceptable impressions sent to the laboratories. It could either be clinicians' factors, material properties factors or patient factors. As for clinicians, whether the lack of knowledge and experience, poor manipulation of the impression material, lack of attention to details, low awareness on the need of critical self-evaluation or even financial constraint could be the possible underlying reasons for these unacceptable impressions being sent to the laboratory.

From the histogram [Figures 3 and 4], it can be seen that polyether (monophase) had high incidence of voids and tears in the impressions. This may be explained by the usual improper technique applied by the clinicians

\begin{tabular}{l} 
Table 2: Criteria for impression quality evaluation \\
\hline Record crucial fine details of tooth preparations \\
Voids (air bubbles) at tooth preparation \\
Tear at finished margin of tooth preparation \\
Flow of impression material
\end{tabular}

with this material. Technique of just loading the tray without using a small syringe to simultaneously apply material at preparation margins resulted in voids and

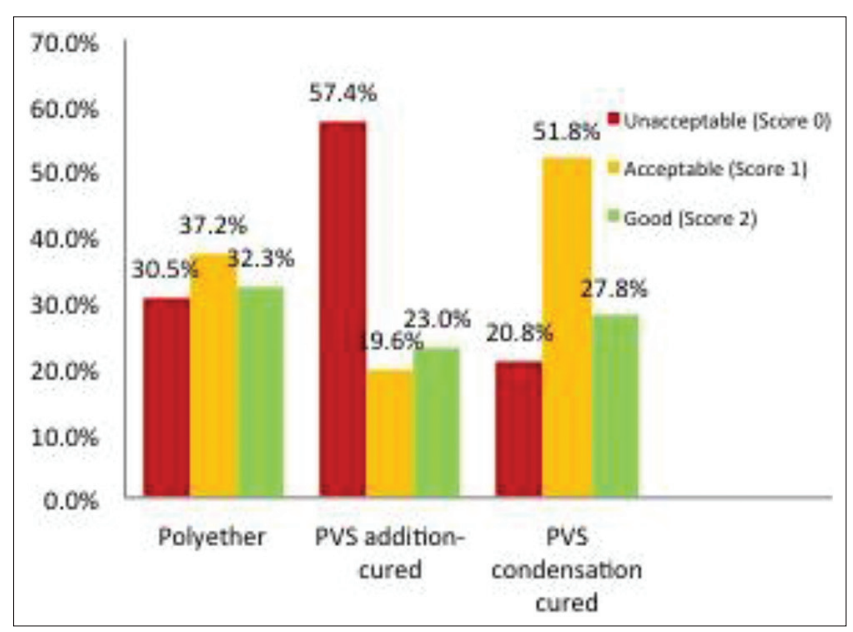

Figure 5: Flow problem in impression

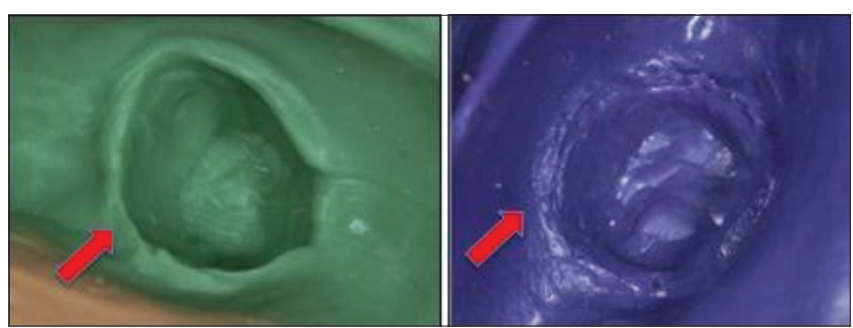

Figure 6: Poor detail of tooth preparation

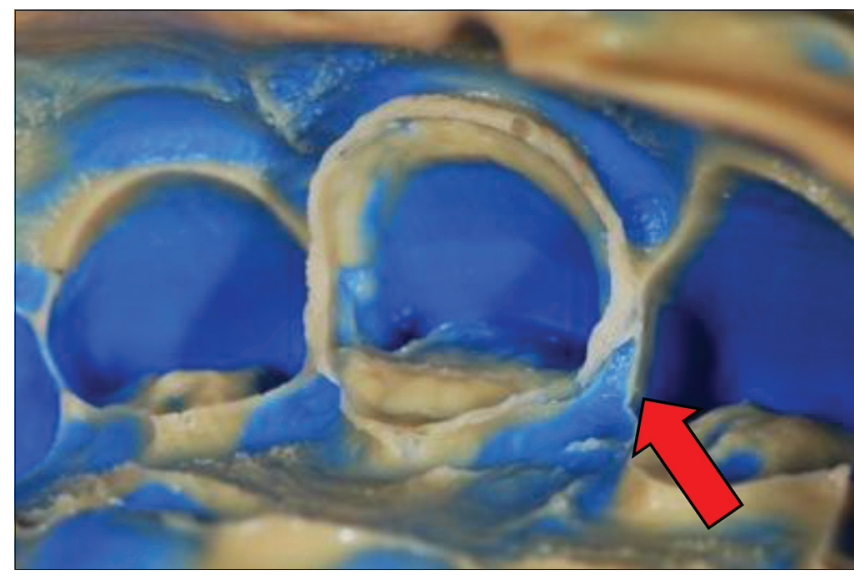

Figure 7: Tear at finished margin
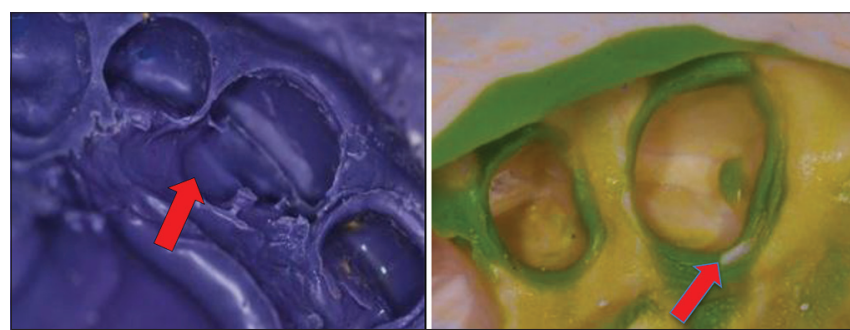

Figure 8: Voids at tooth preparation 
tears formation. As for flow problems [Figure 5], poor manipulation of PVS addition-cured silicones may cause present of streak and steps in the impression. In dual-phase 1-step technique, unexpected early set of the light-body syringed intra-orally due to oral cavity temperature will form steps with the heavy-body loaded in the tray. Insufficient loading of heavy-body impression into the tray was also the common cause of these errors.

Limitation of the study during the evaluation process was the lack of magnification equipment used during examination as magnification might help to provide better examination of the impressions.

\section{CONCLUSION}

The general dental practitioners in Klang Valley areas used reasonable type of trays and impression materials for their crown and bridgework cases. However, within the limitations of this study, we found that the qualities of working impressions sent to the laboratories were unacceptable. The high frequency of detectable errors [Figures 6-8] found in the impressions sent is a serious concern as this will result in poor fitted fixed prostheses provided to patients. A more critical evaluation of impressions by the dentists themselves is recommended. This can be improved by attending short CPD lectures or courses to update their knowledge and skills on current techniques in fixed prosthodontics. These courses may be organized by respected organizations for the general practitioners in the future.

\section{ACKNOWLEDGMENT}

We would like to acknowledge all the dental laboratories owners and staffs where we obtained our impressions sample. This study was funded by Research Acculturation Grant Scheme (600-RMI/RAGS 5/3 (126/2012)) grant from Research Management Institute (RMI), Universiti Teknologi MARA.

\section{REFERENCES}

1. The glossary of prosthodontic terms. The Academy of Prosthodontics. J Prosthet Dent 1999;71:41-112.

2. Piwowarczyk A, Ottl P, Büchler A, Lauer HC, Hoffmann A. In vitro study on the dimensional accuracy of selected materials for monophase elastic impression making. Int J Prosthodont 2002;15:168-74.

3. Ceyhan JA, Johnson GH, Lepe X. The effect of tray selection, viscosity of impression material, and sequence of pour on the accuracy of dies made from dual-arch impressions. J Prosthet Dent 2003;90:143-9.

4. Joshi PR, Bharat GS, Shrenoy V. Comparative evaluation of dimensional accuracy of addition silicone and condensation silicone. Malays Dent J 2009;30:34-42.

5. Thongthammachat S, Moore BK, Barco MT $2^{\text {nd }}$, Hovijitra S, Brown DT, Andres CJ. Dimensional accuracy of dental casts: Influence of tray material, impression material, and time. J Prosthodont 2002;11:98-108.

6. Samet N, Shohat M, Livny A, Weiss EI. A clinical evaluation of fixed partial denture impressions. J Prosthet Dent 2005;94:112-7.

7. O’Brien WJ. Dental Materials and their selection. $3^{\text {rd }}$ ed. Quintessence Publishing Co, Inc; 2002. p. 90-112

8. Ghani SM. The accuracy of impressions made using 'BorderlockMouldable Trays'. Unpublished MClinDent dissertation. University of Edinburgh; 2010.

How to cite this article: Zu Saifudin AA, Kamaruddin F, Ab Ghani SM. The quality of working impressions for the fabrication of fixed prosthodontics prostheses (crown and bridgework). Eur J Gen Dent 2014;3:100-4.

Source of Support: Nil, Conflict of Interest: None declared.

Announcement

\section{iPhone App}

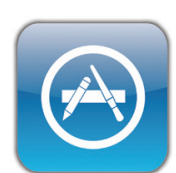

Download

iPhone, iPad application
A free application to browse and search the journal's content is now available for iPhone/iPad. The application provides "Table of Contents" of the latest issues, which are stored on the device for future offline browsing. Internet connection is required to access the back issues and search facility. The application is Compatible with iPhone, iPod touch, and iPad and Requires iOS 3.1 or later. The application can be downloaded from http://itunes.apple.com/us/app/medknow-journals/ $\mathrm{id} 458064375$ ? $\mathrm{s}=1 \& \mathrm{mt}=8$. For suggestions and comments do write back to us. 